\title{
Biospecimen Reporting for Improved Study Quality
}

\author{
Helen M. Moore, Andrea Kelly, ${ }^{1}$ Scott D. Jewell, ${ }^{3}$ Lisa M. McShane, ${ }^{4}$ Douglas P. Clark, ${ }^{5}$ \\ Renata Greenspan, ${ }^{6}$ Pierre Hainaut, ${ }^{7}$ Daniel F. Hayes, ${ }^{8}$ Paula Kim, ${ }^{9}$ Elizabeth Mansfield, ${ }^{10}$ \\ Olga Potapova, ${ }^{11}$ Peter Riegman, ${ }^{12}$ Yaffa Rubinstein, ${ }^{13}$ Edward Seijo, ${ }^{14}$ Stella Somiari, ${ }^{15}$ \\ Peter Watson, ${ }^{16}$ Heinz-Ulrich Weier, ${ }^{17}$ Claire Zhu, ${ }^{18}$ and Jim Vaught ${ }^{1}$
}

Human biospecimens are subject to a number of different collection, processing, and storage factors that can significantly alter their molecular composition and consistency. These biospecimen preanalytical factors, in turn, influence experimental outcomes and the ability to reproduce scientific results. Currently, the extent and type of information specific to the biospecimen preanalytical conditions reported in scientific publications and regulatory submissions varies widely. To improve the quality of research utilizing human tissues, it is critical that information regarding the handling of biospecimens be reported in a thorough, accurate, and standardized manner. The Biospecimen Reporting for Improved Study Quality recommendations outlined herein are intended to apply to any study in which human biospecimens are used. The purpose of reporting these details is to supply others, from researchers to regulators, with more consistent and standardized information to better evaluate, interpret, compare, and reproduce the experimental results. The Biospecimen Reporting for Improved Study Quality guidelines are proposed as an important and timely resource tool to strengthen communication and publications around biospecimen-related research and help reassure patient contributors and the advocacy community that the contributions are valued and respected.

\section{Introduction}

$\mathrm{H}$ UMAN BIOSPECIMENS provide the basis for research leading to better understanding of human disease biology and discovery of new treatments that are tailored to individual patients with cancer or other diseases. These biological materials are subject to a number of different collection, processing, and storage factors that can significantly alter their molecular composition and consistency. These preanalytical factors, in turn, influence experimental outcomes and the ability to reproduce scientific results. ${ }^{1-6}$ Currently, the extent and type of information specific to the biospecimen preanalytical conditions reported in scientific publications and regulatory submissions vary widely. To improve the quality of research utilizing human tissues, it is critical that information regarding the handling of biospecimens be reported in a thorough, accurate, and standardized manner.

The purpose of this article was to make recommendations for the reporting of data elements for human biospecimens, defined as solid tissues and bodily fluids, used in biomedical

\footnotetext{
${ }^{1}$ Office of Biorepositories and Biospecimen Research, National Cancer Institute, National Institutes of Health, Department of Health and Human Services, Rockville, Maryland.

${ }^{2}$ Rose Li and Associates, Inc., Brookeville, Maryland.

${ }^{3}$ Program for Biospecimen Science, Van Andel Research Institute, Grand Rapids, Michigan. ${ }^{4}$ Biometric Research Branch, National Cancer Institute, Rockville, Maryland.

${ }^{5}$ Division of Cytopathology, The Johns Hopkins Hospital, Baltimore, Maryland.

${ }^{6}$ U.S.M.C.I./Walter Reed Army Medical Center, Washington, District of Columbia.

${ }^{7}$ International Agency for Research on Cancer, World Health Organization, Lyon, France.

${ }^{8}$ Breast Oncology Program Stuart B. Padnos Professor in Breast Cancer Research, University of Michigan Comprehensive Cancer Center, Ann Arbor, Michigan.

${ }^{9}$ TRAC-Translating Research Across Communities, Green Cove Springs, Florida.

${ }^{10}$ CDRH-Office of In Vitro Diagnostic Device Evaluation and Safety, Center for Devices and Radiological Health, Silver Spring, Maryland.

${ }^{11}$ Cureline, Inc., South San Francisco, California.

${ }^{12}$ Erasmus MC Tissue Bank, Rotterdam, The Netherlands.

${ }^{13}$ Office of Rare Diseases Research, National Institutes of Health, Rockville, Maryland.

${ }^{14} \mathrm{H}$. Lee Moffitt Cancer Center and Research Institute, Tampa, Florida.

${ }^{15}$ Windber Research Institute, Windber, Pennsylvania.

${ }^{16}$ Department of Pathology and Laboratory Medicine, University of British Columbia, Victoria, British Columbia, Canada.

${ }^{17}$ Lawrence Berkeley National Laboratory, Berkeley, California.

${ }^{18}$ Division of Cancer Prevention, National Cancer Institute, Rockville, Maryland.
} 
studies. Cell lines and biospecimen derivatives such as nucleic acids or proteins, although crucial for biomedical research, are not intended to fall within the scope of these recommendations. The Biospecimen Reporting for Improved Study Quality (BRISQ) recommendations are intended to apply to any study in which human biospecimens are used. This includes biomedical applications such as translational science, biomarker discovery, clinical trials, technology development, and diagnostic-assay and therapeutics development. The recommended data elements would be reported by an author in a journal publication, by a company in a regulatory submission, or by a biorepository distributing biospecimens. It is intended that the list and the elements within it will be interpreted, modified, and applied according to the context of the study being reported. It is also recognized that information corresponding to all data elements may not be available, but at least for some categories (described below) the known or unknown status of these elements should be documented.

The list of data elements discussed includes general information for consistent documentation of classes of biospecimens and factors that might influence the integrity, quality, and/or molecular composition of biospecimens. Reporting the details enumerated in the BRISQ list does not guarantee biospecimen quality and should not be seen as a substitute for empirical quality evaluations. The purpose of reporting these details is to supply others, from researchers to regulatory agencies, with more consistent and standardized information to better evaluate, interpret, compare, and reproduce the experimental results. To maintain consistency with federal regulations on research involving human subjects, information that might enable individual identification of research participants should be withheld.

The BRISQ list has been constructed as an initial step toward defining reporting recommendations. The list will likely evolve as more is learned about the factors that influence biospecimen quality and composition and, in turn, their effects on biospecimen analysis. It is envisioned that future iterations of the BRISQ recommendations might include changes to the list of elements and the relative weight thereof in accordance with evidence-based scientific and medical findings and technological developments.

\section{Materials and Methods}

A half-day workshop, Development of Biospecimen Reporting Criteria for Publications, was held at the National Cancer Institute (NCI) 2009 Biospecimen Research Network Symposium (http://biospecimens.cancer.gov/ meeting/brnsymposium) to initiate a discussion on biospecimen reporting recommendations. Workshop attendees included individuals covering a broad range of expertise: laboratory scientists, clinicians, pathologists, statisticians, patient advocates, biobankers, journal editors, leaders of relevant professional societies, and other stakeholders. The attendees noted that reporting guidelines covering many aspects of biomedical studies already exist, particularly guidelines relevant to experimental design and data reporting. ${ }^{*}$ It was proposed that the BRISQ recommendations apply to all

*The EQUATOR project (http://www.equator-network.org/) provides an extensive listing of guidelines for health research. studies utilizing human biospecimens and thus complement existing guidelines by filling a niche concerning reporting of biospecimen characteristics and preanalytical variables.

The attendees further proposed that the BRISQ recommendations should broadly encompass solid tissues and bodily fluids, rather than including separate lists for these biospecimen types. It was also agreed that a committee to develop biospecimen reporting recommendations should be formed to take the effort forward. Many of the individuals and disciplines participating in the workshop were included when the BRISQ committee was subsequently formed.

Formulation of the recommendations was based on consideration of what biospecimen information could enable a science reviewer to fully evaluate or replicate a reported study. The preliminary list included the most commonly available data elements. The committee considered the characteristics of the biospecimens themselves as well as numerous preanalytical factors. Types of data elements include the tissue type and the pathology of the sample; patient characteristics that might influence the biospecimens, such as vital and disease states; and the collection and handling of the biospecimens, for example, the stabilization, shipping, and storage conditions.

The preliminary list of recommendations was refined by consulting the NCI Biospecimen Research Database (http:// brd.nci.nih.gov), an online resource compiling peer-reviewed articles that address biospecimen science. The Biospecimen Research Database's terminology for scientific literature curation that was deemed relevant was incorporated into the initial BRISQ list. This terminology served as a starting point for discussion at monthly teleconferences by the BRISQ committee.

\section{Results}

The committee composed a list of data elements that represent factors believed to often influence biospecimen quality and thus should be considered for reporting, if known or applicable, for the particular study; for example, some list of elements will be more applicable to biospecimens collected for a disease-specific study than those collected for a population-based biospecimen resource. For clarity, these elements are organized according to the lifecycle of the biospecimen (Fig. 1), which spans the period immediately prior to removal from the patient through use in a scientific analysis.

Many reporting elements were discussed, but only some were approved by consensus for inclusion in the guidelines. The committee was mindful that certain information, although important to report, may not have direct relevance to the biology or condition of the biospecimen and, therefore, would not be under the purview of the BRISQ recommendations. The committee attempted to carefully balance scientific interest in having access to extensive data about biospecimen collection, processing, and storage against practical challenges in obtaining such detailed information. Each reporting element included in the guidelines is backed by evidence that the factor could have an effect on the structural integrity and molecular characteristics of the biospecimen or on the ability to perform certain assays on the biospecimen and obtain reliable results. Although the committee recognizes that collection of data about biospecimens can increase the operational costs to collect and use biospecimens, cost was not factored into the exclusion of data elements that were or should be considered necessary. 


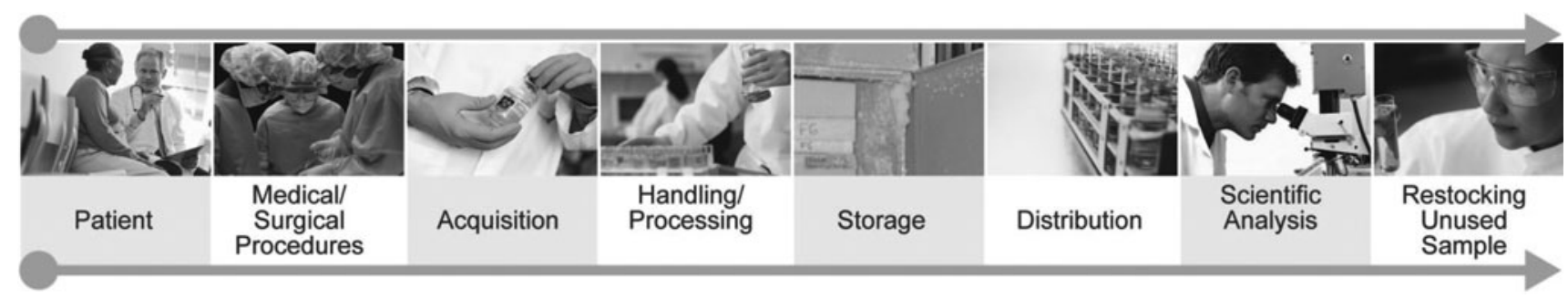

FIG. 1. The lifecycle of the biospecimen. The preanalytical phase of the lifecycle of the biospecimen includes each stage from patient to distribution. Preanalytical variables are addressed in the Biospecimen Reporting for Improved Study Quality list.

The elements in the BRISQ list are prioritized into 3 tiers according to the relative importance of their being reported. The first tier, items recommended to report, includes information such as the organ(s) or the anatomical site from which the biospecimens were derived and the manner in which the biospecimens were collected, stabilized, and preserved; for quick reference, these items are summarized in Table 1. Reporting these items need not be onerous. For example, Beatty et al. included most BRISQ Tier 1 items in the following excerpts:

- "Fine-needle aspiration specimens were obtained from 55 surgically removed specimens of breast cancer within
$1 \mathrm{~h}$ of resection, before tissue fixation. The aspirates were obtained using a 22- to 25-gauge needle and spread directly on slides and fixed in ethanol or formalin or placed in CytoLyt for preparation of ThinPrep slides according to the manufacturer's protocol. Corresponding formalin-fixed, paraffin-embedded tissue specimens were fixed in $10 \%$ neutral buffered formalin for $18-24 \mathrm{~h}$ according to routine procedures and embedded in paraffin."

- "All fine-needle aspiration cytologic slides were air dried and stored at room temperature before fluorescence in situ hybridization analysis."

Table 1. Quick-Reference Biospecimen Reporting for Improved Study Quality Summary/Checklist: Tier 1 Items to Report if Known and Applicable

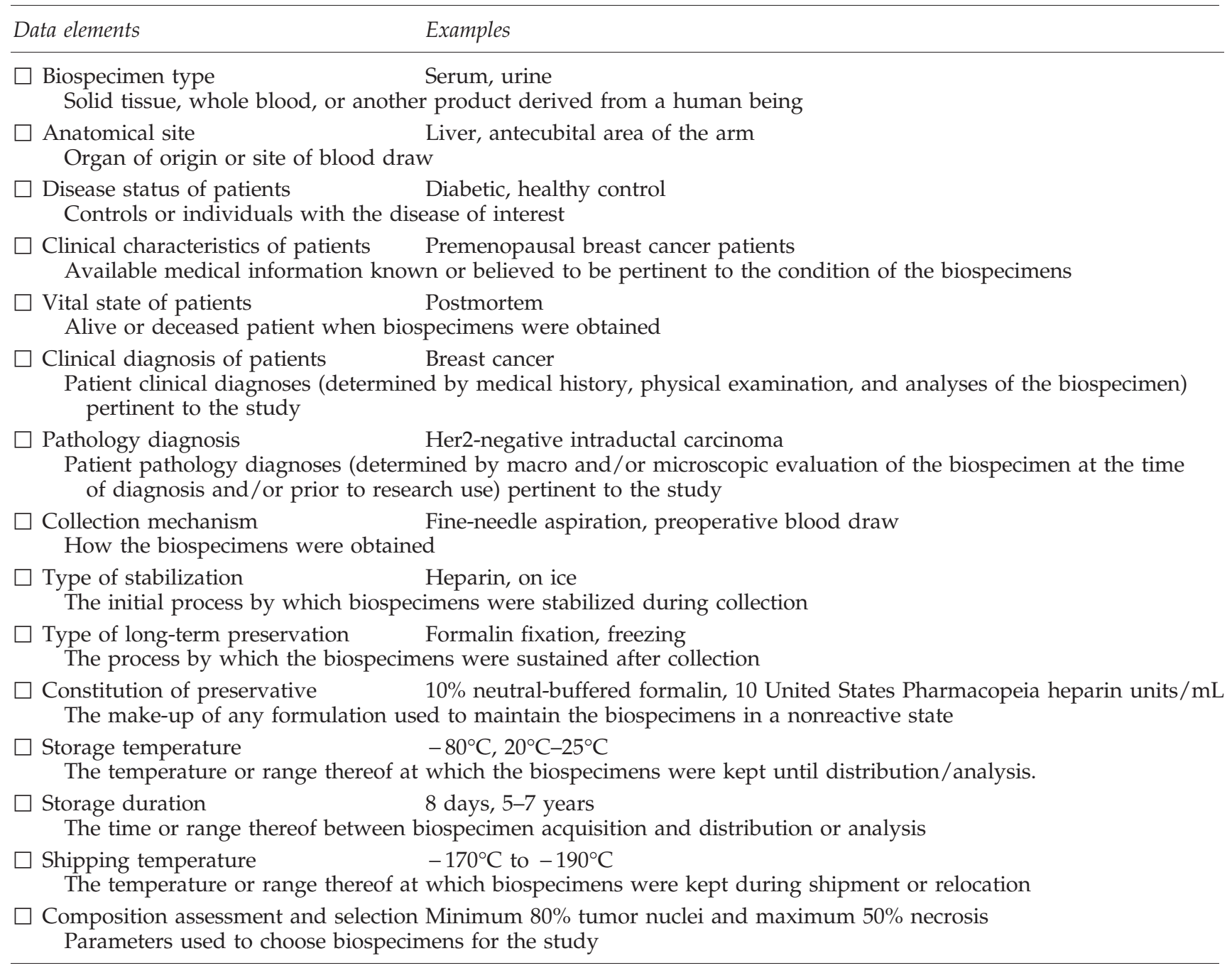


Items beneficial to report form the second tier. These are data elements an evaluator might find helpful to know but may be slightly less crucial to the scientific contribution or less likely to be annotated, such as the time from biospecimen excision/acquisition to stabilization.

Additional items to report compose the third tier. These include information about conditions that might be useful to know concerning the biospecimens but are not known to be as likely to influence research results or are unlikely to be available to researchers, such as environmental factors to which patients were exposed or the type of storage container in which the biospecimens were kept.

The full BRISQ list featured in Table 2 includes each item and its definition along with additional columns that were designed for an author or reviewer to track where the listed items are reported for a particular study. To the right of the Item descriptions is a column assigning each item a unique Roman numeral/letter/number identification code. The far right column provides space to note where each item may be found in a manuscript or application. The far left Apply to column indicates whether the BRISQ item is applicable to "all" biospecimen types or is more appropriate for solid "tissue" biospecimens or "fluid" biospecimens (such as blood, urine, or other fluids). For example, item III.b, "Type of long-term preservation," is pertinent to all types of biospecimens; item III.b.2, "Time in fixative/preservative solution," is more relevant to solid tissue than to fluid biospecimens; and item III.c, "Aliquot volume," applies more often to fluid than to solid tissue biospecimens. See the Appendix for examples of prior studies, with examples of the effect of each BRISQ data element.

When reporting elements of the BRISQ list, standard operating procedures specifying many of the pertinent details, such as blood collection protocols, may be provided or referenced; any referenced documents should be publicly available. It is preferable that most Tier 1 items relevant to the biospecimen and particular scientific study be reported directly in the intended publication rather than be cited from another document. Detailed descriptions that are too lengthy to be accommodated should be made available as supplemental materials online. Whether the laboratory performing the study was operating under any formal certification or accreditation should be stated if applicable to the study being reported.

The BRISQ committee discussed whether to request information that the biorepository and/or researcher had obtained ethical clearance to collect the biospecimens and perform the study. Clearance from an institutional review board or similar body is important to report in publications, and its reporting is generally required by journals. However, it is not immediately pertinent to the structural integrity and molecular characteristics of the biospecimen and is thus not included in the BRISQ recommendations. Similarly, accurate biospecimen-tracking mechanisms are essential to biobanking but not immediately pertinent to the condition of the biospecimen and are thus also not included in the BRISQ data elements list.

Surgical parameters, such as type of anesthesia or receipt of blood or other intraoperative infusates, were recognized to be of potential significance to the condition of the biospecimens. However, these data are often not known. When it is available, information about anesthesia and intraoperative treatments that may influence the condition of the biospecimens should be reported. These elements were not included in the BRISQ list because currently such information is rarely available or not required to be recorded as part of biospecimen collection efforts. If or when surgical parameters are determined to be critical through systematic biospecimen research studies, these elements will be integrated into future recommendations.

Several preservation parameters known to influence the condition of biospecimens and the results of analyses have been included in the list of recommendations. Researchers should state the rationale for the chosen preservation parameters. For example, if the type and temperature of the biospecimen preservative were selected to optimize stability, extraction, and analysis of a particular analyte, this should be mentioned.

The BRISQ committee recognized the need for greater specificity in the anatomic and histologic details reported concerning solid tissue biospecimens. The committee agreed that the level of detail with which pathology characteristics are reported should be enough to sufficiently address the scientific research question. These characteristics include not only the tissue site of the biospecimen and the relation of the biospecimen to the pertinent clinical diagnosis within the tissue site, but also the composition and pathology within the biospecimen where relevant.

The BRISQ committee included members of the NCI Office of Biorepositories and Biospecimen research (OBBR), participants from the OBBR Biospecimen Research Network Symposium, and members of the International Society for Biological and Environmental Repositories (ISBER) and the committees responsible for the REporting recommendations for tumor MARKer prognostic studies (REMARK) ${ }^{8}$ and STrengthening the Reporting of OBservational studies in Epidemiology (STROBE) ${ }^{9}$ guidelines. Essential harmonization with similar efforts are underway by these groups.

\section{Discussion}

An adage in the business community states, "That which is measured improves. That which is measured and reported improves exponentially." The BRISQ reporting recommendations represent the product of extensive discussion and input from researchers with varied types of expertise and from many stakeholders, all of whom share the common goal of improving biospecimen reporting and, by extension, fields in which biospecimens are employed. The committee believes that by providing details concerning preanalytical factors that might affect assay results, investigators will further improve the quality of biomedical studies, including research for developing cancer biomarkers for screening, early detection, and treatment.

Adoption of the BRISQ recommendations is expected to help authors, reviewers, editors, and regulatory officials evaluate whether sufficient information about the biospecimens has been provided to enable assessment of the influence of preanalytical biospecimen factors on study results. If reported, this information will allow improved evaluation, interpretation, comparison, and reproduction of the results from studies that employ human biospecimens. Although items in any tier might not be available or items in Tiers 2 or 3 might not be considered significant to report, increased awareness of their potential influence on biospecimen studies might lead to improved tracking and reporting in the future.

The BRISQ recommendations may be implemented by anyone reporting on studies involving biospecimens. 


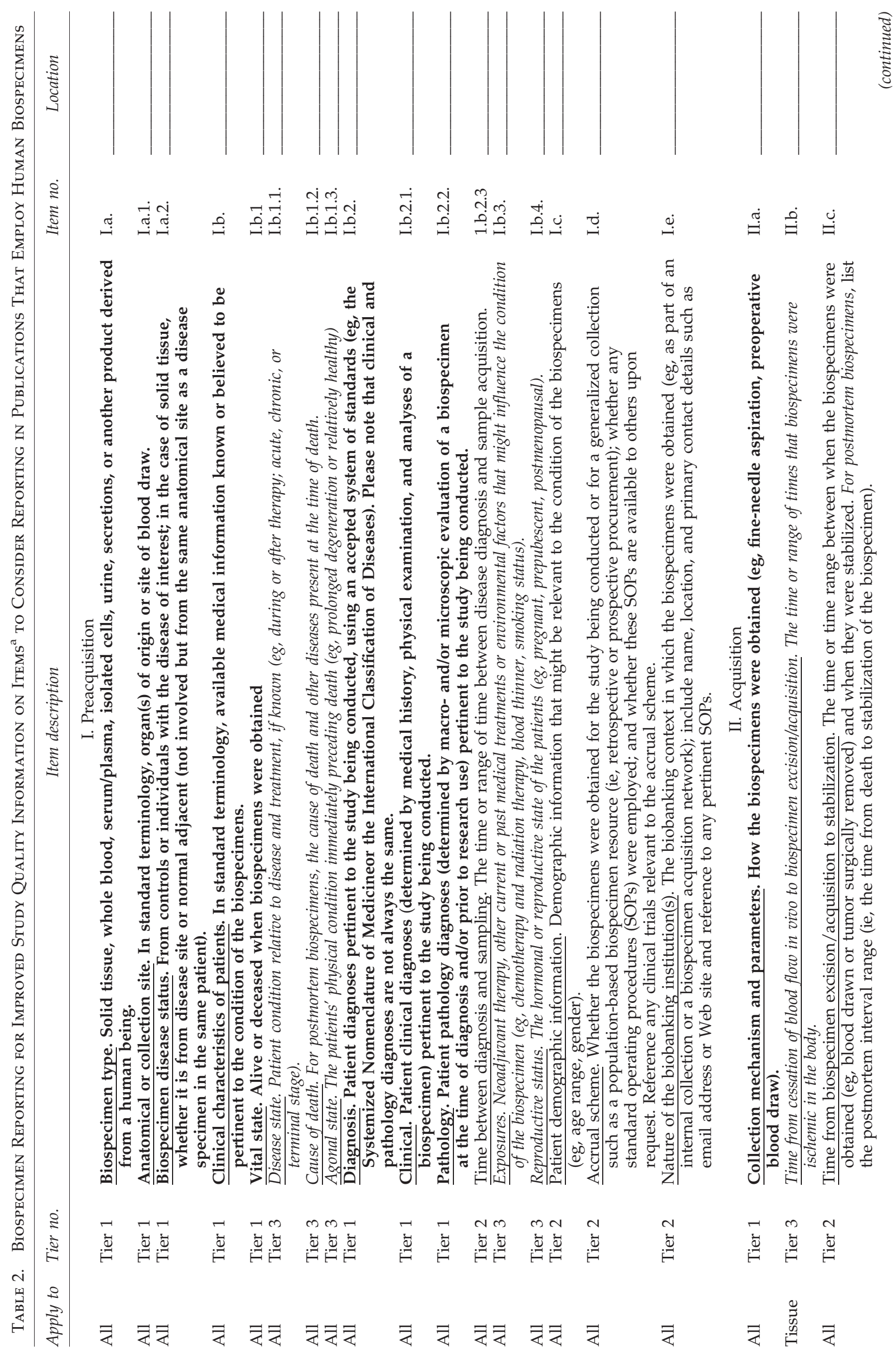




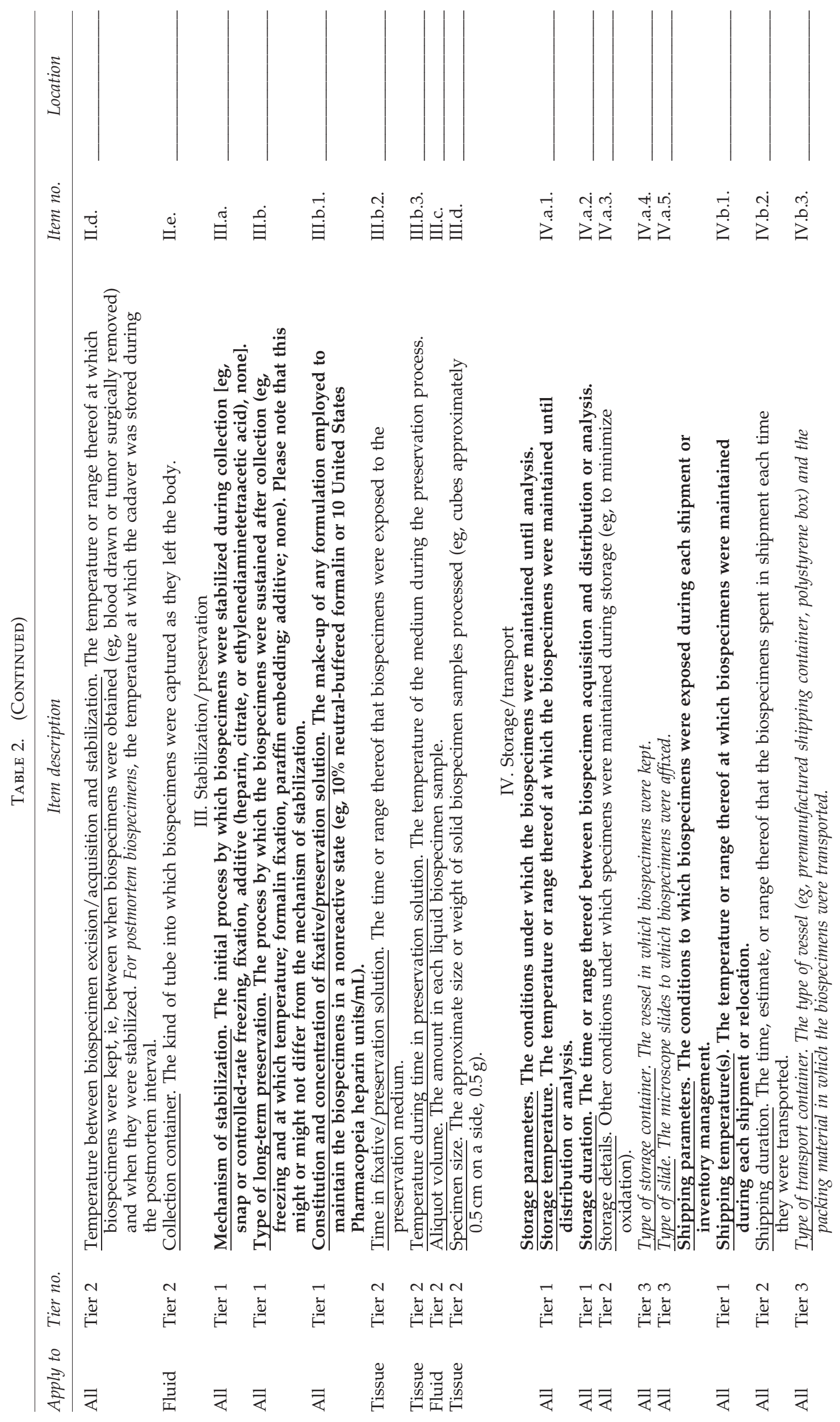




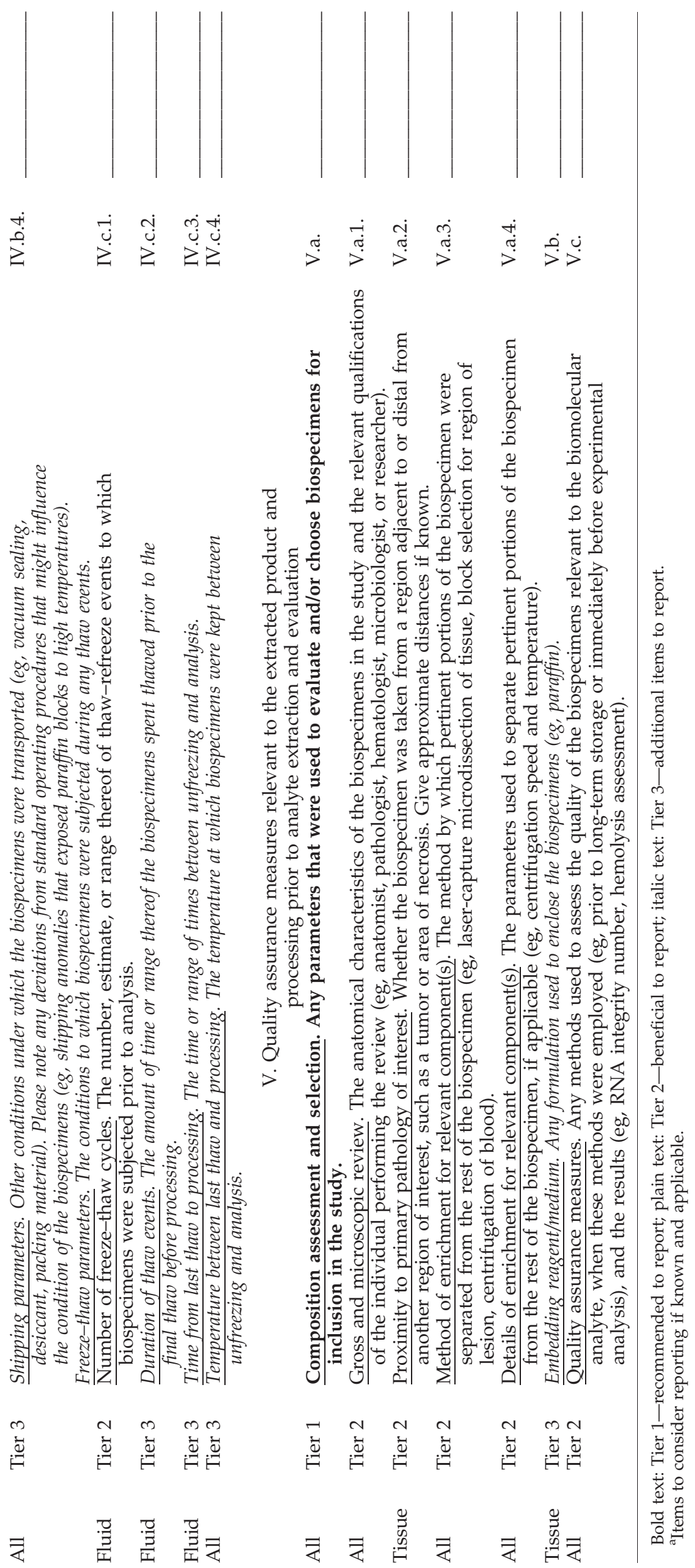


Reviewers, editors, and regulatory officials might also employ the list as a tool for evaluating whether sufficient biospecimen information has been included in a manuscript or application. In addition, the recommendations might be employed by investigators requesting biospecimens from a biospecimen resource: essential items on the list might be checked off to indicate that they are required annotation for the desired samples. Elements of BRISQ that document preanalytical variables for tissue biospecimens could be economically captured using a reporting system such as the Standard PREanalytical Code, or SPREC, which was recently published by the ISBER Working Group on Biospecimen Science. $^{10}$

BRISQ reporting items will not be necessarily applicable to every study, and authors and reviewers are urged to use their judgment to decide which factors are essential. It is not always possible for investigators to ascertain every recommended element for every biospecimen, even for Tier 1 items, but unknown elements relevant to the study being reported should be fully acknowledged with a discussion of possible implications that the missing information might have on the study conclusions. Unknown or unreported Tier 1 data elements should not be considered a reason for automatic dismissal of a report or conditional for the award of a grant. The final decision on acceptability of missing Tier 1 information should be specific to the study context.

When consulting the BRISQ list, researchers should evaluate the importance of each item in the context of the study and adjust their reporting accordingly. An item such as "method of enrichment for relevant components," listed here as Tier 2, might-for example, in the context of a study comparing the efficacy of various enrichment methods-be essential to report and should thus be considered Tier 1 for that study. The converse may also be true, when, for example, an item listed here as Tier 2-such as "temperature between acquisition and stabilization"-is less pertinent to the study at hand-perhaps because the time at this temperature was negligible-and should thus be considered Tier 3.

It is hoped that consideration of the BRISQ recommendations will sensitize the biobanking and research communities and their funding agencies to the importance of tracking preanalytical variables, leading to more judicious selection and handling of experimental human specimens and thus improved study quality. Anecdotally, recommendations such as REMARK seem to have had the effect of spurring researchers to consider the recommendations in advance of conducting their investigations, with the result that researchers might take greater care in the design, conduct, and analysis of their studies. The BRISQ committee envisions a similar trajectory for preanalytical biospecimen data elements. Thus, not only might overall quality of publications improve, but the quality of human biospecimendependent investigation in general might improve over time with the formation and adoption of publication recommendations. It is anticipated that biospecimen resources might use these recommendations to improve on their existing standard operating procedures and annotation thereof. Such improvements could include the acquisition of additional relevant biospecimen data based on the BRISQ recommendations and the release of all such data to researchers as a standard procedure. In this way, biospecimen resources might become major players in the universal application of these recommendations.

Patient contribution of biospecimens for research is a voluntary, generous action aimed at helping advance scientific discovery and progress. The research team, pathologist, and biorepository systems, as the stewards of these biospecimens, have a responsibility to be vigilant and persistent in using methods and practices that protect and preserve the highest possible quality biospecimen and associated data. The BRISQ guidelines are proposed as an important and timely resource tool to strengthen communication and publications around biospecimen-related research and help reassure patient contributors and the advocacy community that the contributions are valued and respected. Researchers are further encouraged to strengthen public outreach and education around the use and potential of human biospecimens ${ }^{11}$ and the biorepository community as these are emerging and potentially misunderstood areas.

\section{Acknowledgment}

This project has been funded in whole or in part with Federal Funds from the NCI, National Institutes of Health, under contract no. HHSN261200800001E and by NIH grant CA136685 (HUW) carried out at the Lawrence Berkeley National Laboratory under contract DE-AC002-05CH11231. The content of this publication does not necessarily reflect the views or policies of the Department of Health and $\mathrm{Hu}-$ man Services, and mention of trade names, commercial products, or organizations does not imply endorsement by the U.S. Government.

\section{Author Disclosure Statement}

No competing financial interests exist.

\section{References}

1. Srinivasan M, Sedmak D, Jewell S. Effect of fixatives and tissue processing on the content and integrity of nucleic acids. Am J Pathol 2002;161:1961-1971.

2. Moore HM, Compton, CC, Lim MD, et al. Biospecimen Research Network Symposium: Advancing Cancer Research through Biospecimen Science. Cancer Res 2009;69:6770-6772.

3. Apweiler R, Aslanidis C, Deufel T, et al. Approaching clinical proteomics: current state and future fields of application in cellular proteomics. Cytometry A 2009;75:816-832.

4. Apweiler R, Aslanidis C, Deufel T, et al. Approaching clinical proteomics: current state and future fields of application in fluid proteomics. Clin Chem Lab Med 2009;47:724-744.

5. Espina V, Muelle C, Edmiston $K$, et al. Tissue is alive: new technologies are needed to address the problems of protein biomarker pre-analytical variability. Proteomics Clin Appl 2009;3:874-882.

6. Ransohoff DF, Gourlay ML. Sources of bias in specimens for research about molecular markers for cancer. J Clin Oncol 2010;28:698-704.

7. Beatty BG, Bryant R, Wang W, et al. HER-2/neu detection in fine-needle aspirates of breast cancer: fluorescence in situ hybridization and immunocytochemical analysis. Am J Clin Pathol 2004;122:246-255

8. McShane LM, Altman DG, Sauerbrei W, et al. Statistics Subcommittee of the NCI-EORTC Working Group on Cancer Diagnostics. Reporting recommendations for tumor marker 
prognostic studies (REMARK). J Natl Cancer Inst 2005;97:11801184.

9. von Elm E, Altman DG, Egger $\mathrm{M}$, et al. STROBE Initiative. The Strengthening the Reporting of Observational Studies in Epidemiology (STROBE) statement: guidelines for reporting observational studies. J Clin Epidemiol 2008;61:344-349.

10. Betsou F, Lehmann S, Ashton G, et al. Standard preanalytical coding for biospecimens: defining the sample PREanalytical code. Cancer Epidemiol Biomarkers Prev 2010;19:1004-1011.

11. Secko DM, Preto N, Niemeyer S, et al. Informed consent in biobank research: a deliberative approach to the debate. Soc Sci Med 2009;68:781-789.
Address correspondence to: Dr. Jim Vaught Office of Biorepositories and Biospecimen Research National Cancer Institute 11400 Rockville Pike Suite 700 Rockville, MD 20852

E-mail: vaughtj@mail.nih.gov

Received 27 December, 2010/Accepted 11 January, 2010 


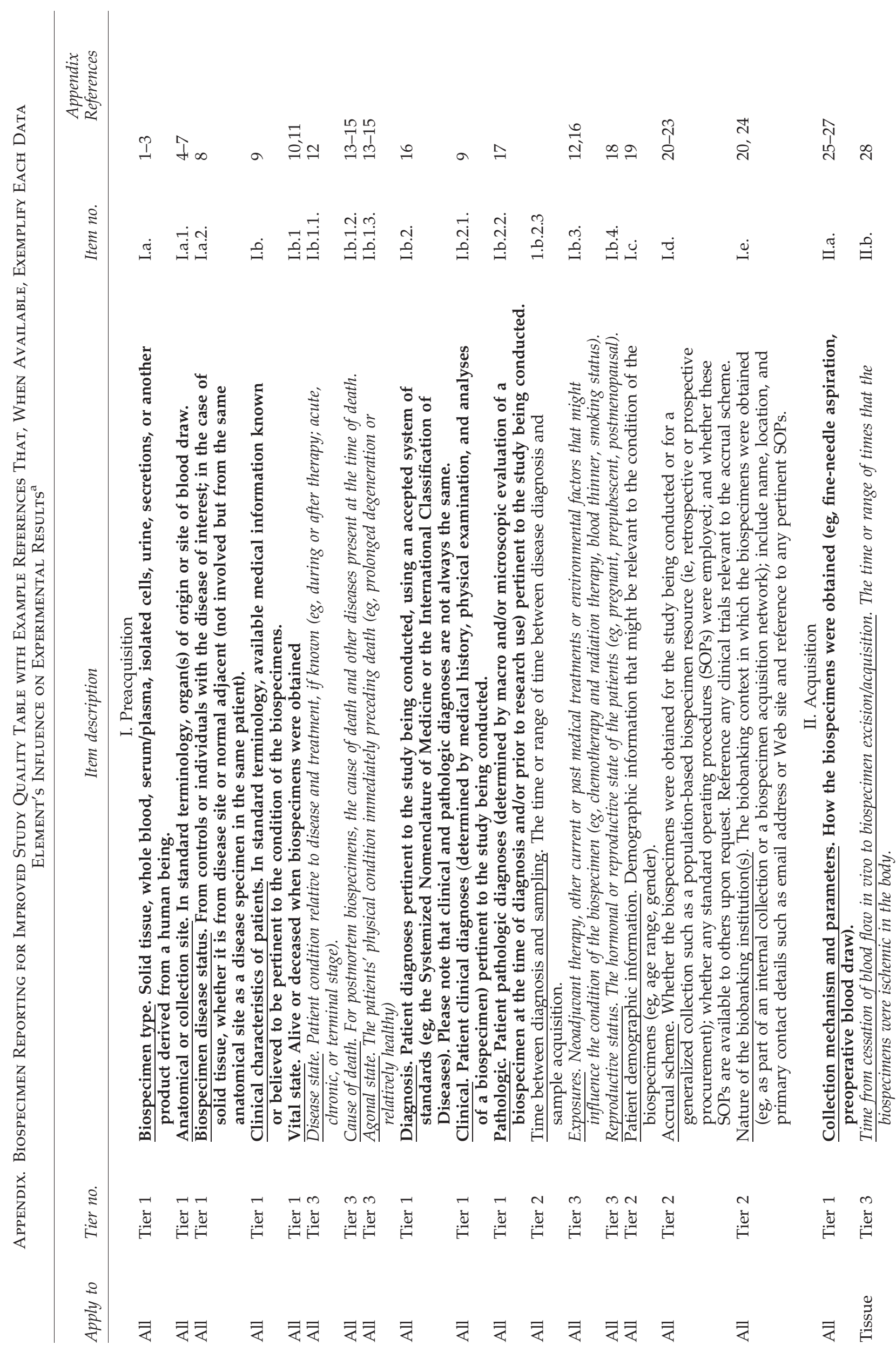




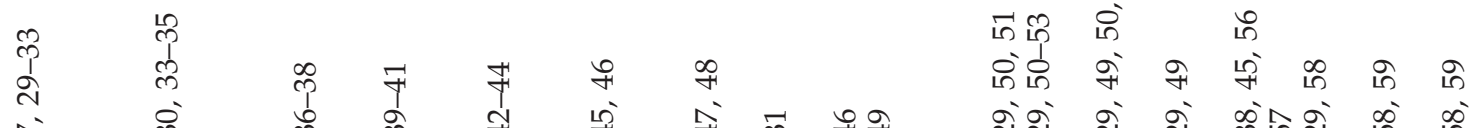

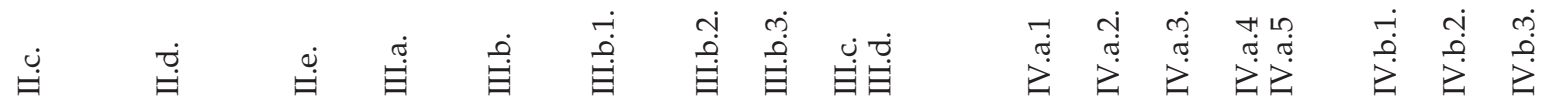

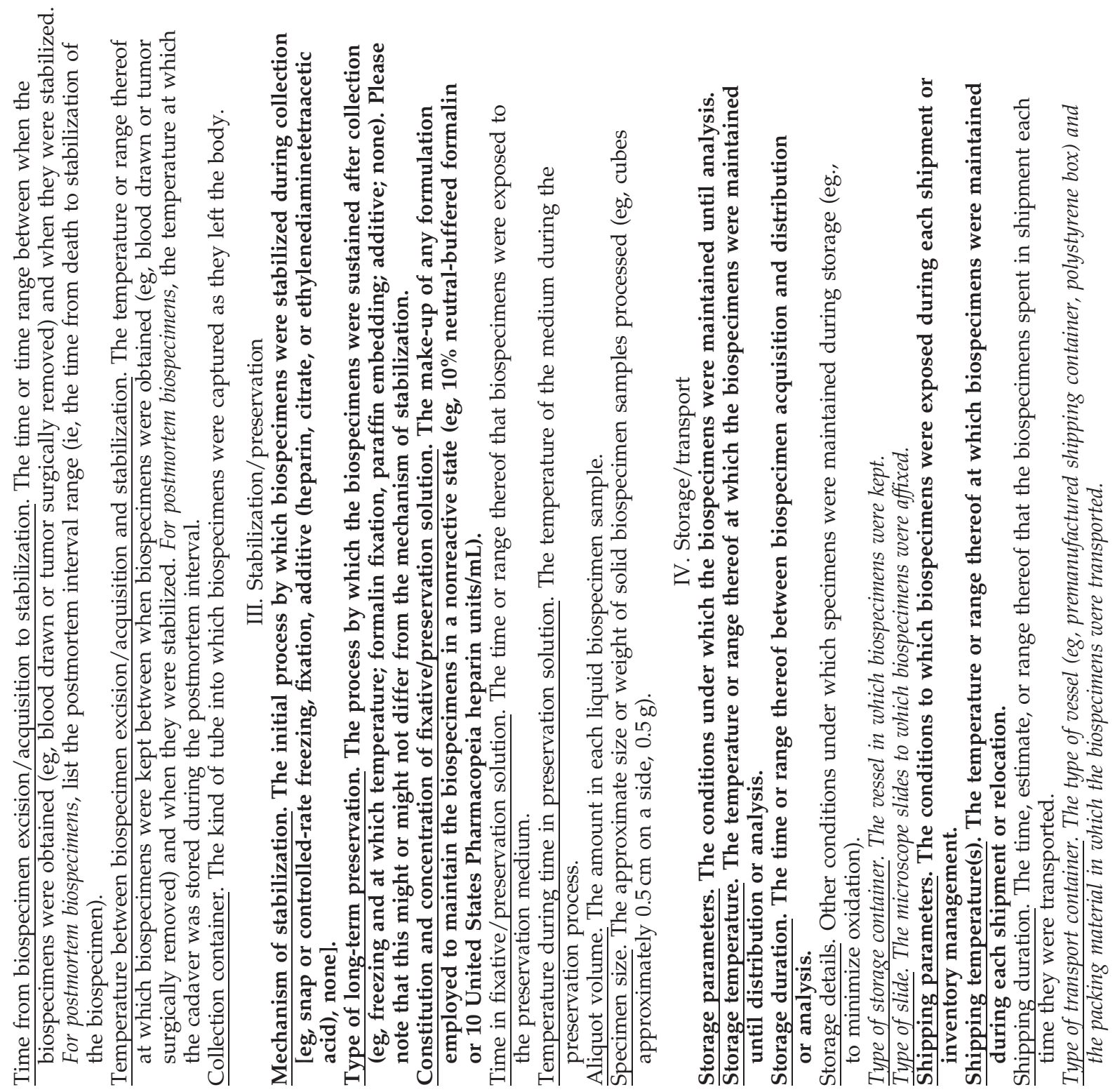

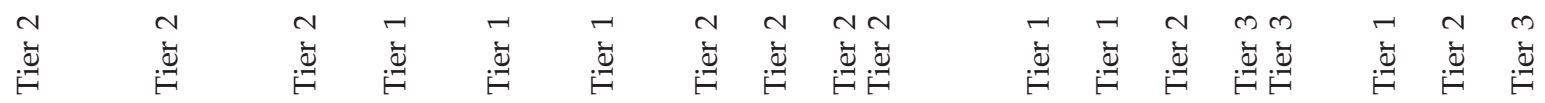

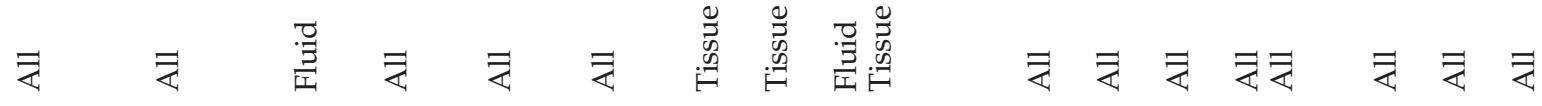




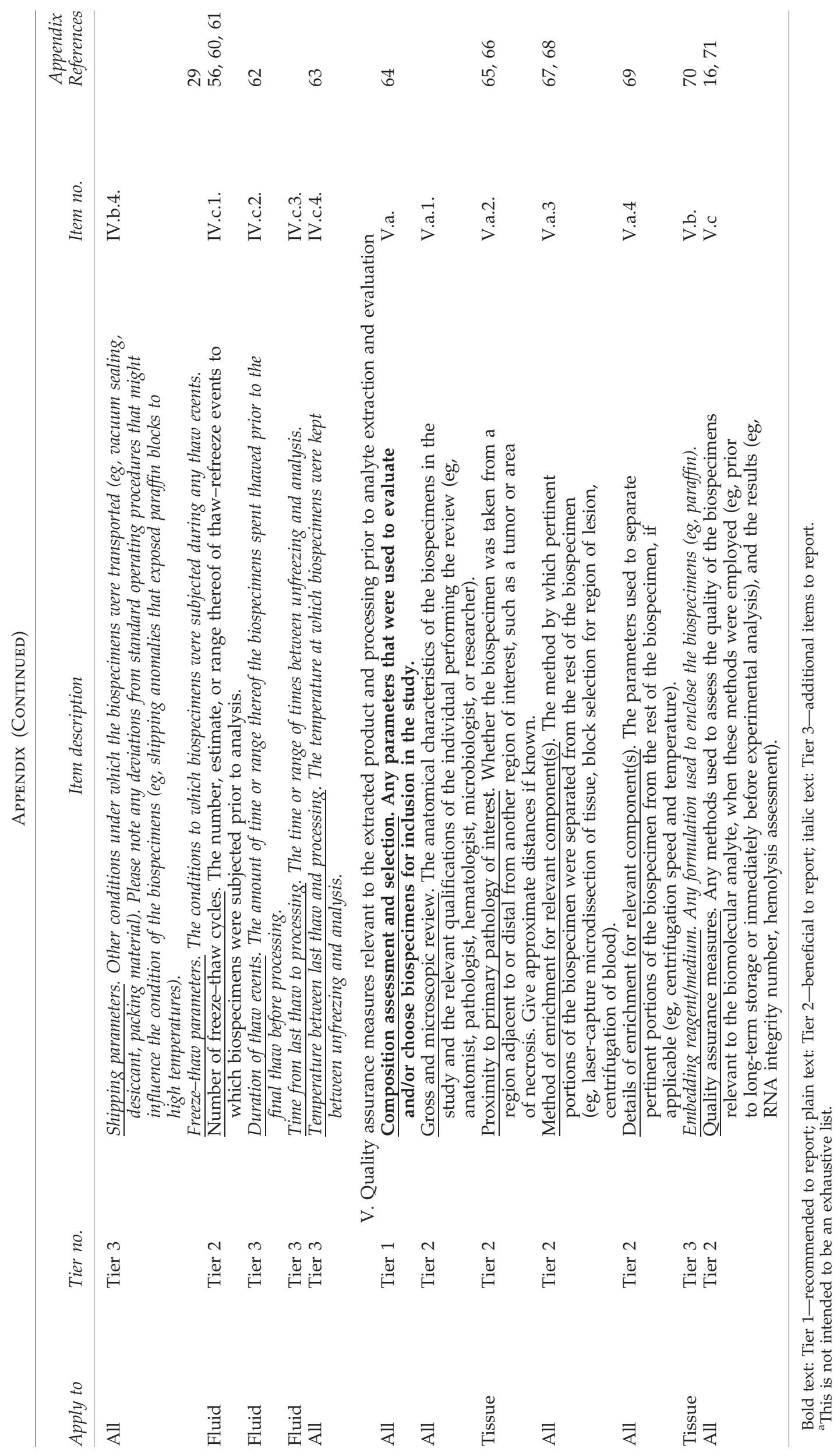




\section{Appendix References}

1. Di Nunno N, Costantinides F, Cina SJ, et al. What is the best sample for determining the early postmortem period by on-thespot flow cytometry analysis? Am J Forensic Med Pathol 2002;23:173-180.

2. Humphreys-Beher MG, King FK, Bunnel B, et al. Isolation of biologically active RNA from human autopsy for the study of cystic fibrosis. Biotechnol Appl Biochem 1986;8:392-403.

3. Barton RH, Nicholson JK, Elliott P, et al. High-throughput $1 \mathrm{H}$ NMR-based metabolic analysis of human serum and urine for large-scale epidemiological studies: validation study. Int J Epidemiol 2008;37 Suppl 1:i31-i40.

4. Centeno BA, Enkemann SA, Coppola D, et al. Classification of human tumors using gene expression profiles obtained after microarray analysis of fine-needle aspiration biopsy samples. Cancer 2005;105:101-109.

5. Hoff-Olsen P, Jacobsen S, Mevåg B, et al. Microsatellite stability in human post-mortem tissues. Forensic Sci Int 2001;119:273-278.

6. Yang ZW, Yang SH, Chen L, et al. Comparison of blood counts in venous, fingertip and arterial blood and their measurement variation. Clin Lab Haematol 2001;23:155-159.

7. Heinrich M, Matt K, Lutz-Bonengel S, et al. Successful RNA extraction from various human postmortem tissues. Int J Legal Med 2007;121:136-142.

8. Weis S, Llenos IC, Dulay JR, et al. Quality control for microarray analysis of human brain samples: the impact of postmortem factors, RNA characteristics, and histopathology. J Neurosci Methods 2007;165:198-209.

9. Tantipaiboonwong P, Sinchaikul S, Sriyam S, et al. Different techniques for urinary protein analysis of normal and lung cancer patients. Proteomics 2005;5:1140-1149.

10. He S, Wang Q, He J, et al. Proteomic analysis and comparison of the biopsy and autopsy specimen of human brain temporal lobe. Proteomics 2006;6:4987-4996.

11. Jones RF, Sunheimer R, Friedman H, et al. Comparison of anteand post-mortem PSA levels for epidemiological studies. Anticancer Res 2005;25(2B):1263-1267.

12. Pinder SE, Provenzano E, Earl H, Ellis IO. Laboratory handling and histology reporting of breast specimens from patients who have received neoadjuvant chemotherapy. Histopathology 2007;50:409-417.

13. Tomita H, Vawter MP, Walsh DM, et al. Effect of agonal and postmortem factors on gene expression profile: quality control in microarray analyses of postmortem human brain. Biol Psychiatry 2004;55:346-352.

14. Preece P, Virley DJ, Costandi M, et al. An optimistic view for quantifying mRNA in post-mortem human brain. Brain Res Mol Brain Res 2003;116:7-16.

15. Johnston NL, Cervenak J, Shore AD, et al. Multivariate analysis of RNA levels from postmortem human brains as measured by three different methods of RT-PCR. Stanley Neuropathology Consortium. J Neurosci Methods 1997;77:83-92.

16. Webster MJ. Tissue preparation and banking. Prog Brain Res 2006;158:3-14.

17. Ellis M, Davis N, Coop A, et al. Development and validation of a method for using breast core needle biopsies for gene expression microarray analyses. Clin Cancer Res 2002;8:1155-1166.

18. Reyna R, Traynor KD, Hines G. et al. Repeated freezing and thawing does not generally alter assay results for several commonly studied reproductive hormones. Fertil Steril 2001;76:823-825.

19. Papale M, Pedicillo MC, Thatcher BJ, et al. Urine profiling by SELDI-TOF/MS: monitoring of the critical steps in sample collection, handling and analysis. J Chromatogr B Analyt Technol Biomed Life Sci 2007;856:205-213.

20. Barnes RO, Parisien M, Murphy LC, et al. Influence of evolution in tumor biobanking on the interpretation of translational research. Cancer Epidemiol Biomarkers Prev 2008;17:3344-3350.
21. McIntosh M, Anderson G, Drescher C, et al. Ovarian cancer early detection claims are biased. Clin Cancer Res 2008;14:7574.

22. Sidiropoulos N, Dumont LJ, Golding AC, et al. Quality improvement by standardization of procurement and processing of thyroid fine-needle aspirates in the absence of on-site cytological evaluation. Thyroid 2009;19:1049-1052.

23. Thorpe JD, Duan X, Forrest R, et al. Effects of blood collection conditions on ovarian cancer serum markers. PLoS One 2007; 2:e1281

24. Karsan A, Eigl BJ, Flibotte S, et al. Analytical and preanalytical biases in serum proteomic pattern analysis for breast cancer diagnosis. Clin Chem 2005;51:1525-1528.

25. Sung MT, Lin H, Koch MO, et al. Radial distance of extraprostatic extension measured by ocular micrometer is an independent predictor of prostate-specific antigen recurrence: a new proposal for the substaging of pT3a prostate cancer. Am J Surg Pathol 2007;31:311-318.

26. Morrison C, Palatini J, Riggenbach J, et al. Fine-needle aspiration biopsy of non-Hodgkin lymphoma for use in expression microarray analysis. Cancer 2006;108:311-318.

27. Schaub S, Wilkins J, Weiler T, et al. Urine protein profiling with surface-enhanced laser-desorption/ionization time-of-flight mass spectrometry. Kidney Int 2004;65:323-332.

28. Smith JL, Pillay SP, de Jersey J, et al. Effect of ischaemia on the activities of human hepatic acyl-CoA:cholesterol acyltransferase and other microsomal enzymes. Clin Chim Acta 1989;184:259268.

29. Visvikis S, Schlenck A, Maurice M. DNA extraction and stability for epidemiological studies. Clin Chem Lab Med. 1998;36:551555.

30. Micke P, Ohshima M, Tahmasebpoor S, et al. Biobanking of fresh frozen tissue: RNA is stable in nonfixed surgical specimens. Lab Invest 2006;86:202-211.

31. Burke WJ, O'Malley KL, Chung HD, et al. Effect of pre- and postmortem variables on specific mRNA levels in human brain. Brain Res Mol Brain Res 1991;11:37.

32. Spruessel A, Steimann G, Jung M, et al. Tissue ischemia time affects gene and protein expression patterns within minutes following surgical tumor excision. Biotechniques 2004;36:10301037.

33. Espina V, Edmiston $\mathrm{KH}$, Heiby $\mathrm{M}$, et al. A portrait of tissue phosphoprotein stability in the clinical tissue procurement process. Mol Cell Proteomics 2008;7:1998-2018.

34. van Maldegem F, de Wit M, Morsink F, et al. Effects of processing delay, formalin fixation, and immunohistochemistry on RNA Recovery From Formalin-fixed Paraffin-embedded Tissue Sections. Diagn Mol Pathol 2008;17:51-58.

35. Langebrake C, Günther K, Lauber J, et al. Preanalytical mRNA stabilization of whole bone marrow samples. Clin Chem 2007;53: 587-593.

36. Yucel A, Karakus R, Cemalettin A. Effect of blood collection tube types on the measurement of human epidermal growth factor. J Immunoassay Immunochem 2007;28:47-60.

37. Drake SK, Bowen RA, Remaley AT, et al. Potential interferences from blood collection tubes in mass spectrometric analyses of serum polypeptides. Clin Chem 2004;50:2398-2401.

38. Preissner CM, Reilly WM, Cyr RC, et al. Plastic versus glass tubes: effects on analytical performance of selected serum and plasma hormone assays. Clin Chem 2004;50:1245-1247.

39. Frank M, Döring C, Metzler D, et al. Global gene expression profiling of formalin-fixed paraffin-embedded tumor samples: a comparison to snap-frozen material using oligonucleotide microarrays. Virchows Arch 2007;450:699-711.

40. Scicchitano MS, Dalmas DA, Bertiaux MA, et al. Preliminary comparison of quantity, quality, and microarray performance of RNA extracted from formalin-fixed, paraffin-embedded, and unfixed frozen tissue samples. J Histochem Cytochem 2006;54: 1229-1237. 
41. Narayanan S. Considerations in the application of selected molecular biology techniques in the clinical laboratory: preanalytical and analytical issues. Rinsho Byori 1996;Suppl 103: 262-270.

42. Greer CE, Lund JK, Manos MM. PCR amplification from paraffin-embedded tissues: recommendations on fixatives for longterm storage and prospective studies. PCR Methods Appl 1991;1:46-50.

43. Beatty BG, Bryant R, Wang W, et al. HER-2/neu detection in fine-needle aspirates of breast cancer: fluorescence in situ hybridization and immunocytochemical analysis. Am J Clin Pathol 2004;122:246-255.

44. Kouri T, Malminiemi O, Penders J. Limits of preservation of samples for urine strip tests and particle counting. Clin Chem Lab Med 2008;46:703-713.

45. Zsikla V, Baumann M, Cathomas G. Effect of buffered formalin on amplification of DNA from paraffin wax embedded small biopsies using real-time PCR. J Clin Pathol 2004;57:654-656.

46. Ferry JD, Collins S, Sykes E. Effect of serum volume and time of exposure to gel barrier tubes on results for progesterone by Roche Diagnostics Elecsys 2010. Clin Chem 1999;45:15741575.

47. Macabeo-Ong M, Ginzinger DG, Dekker N, et al. Effect of duration of fixation on quantitative reverse transcription polymerase chain reaction analyses. Mod Pathol 2002;15:979-987.

48. Miething F, Hering S, Hanschke B, et al. Effect of fixation to the degradation of nuclear and mitochondrial DNA in different tissues. J Histochem Cytochem 2006;54:371-374.

49. Gillio-Tos A, De Marco L, Fiano V, et al. Efficient DNA extraction from 25-year-old paraffin-embedded tissues: study of 365 samples. Pathology 2007;39:345-348.

50. Sigurdson AJ, Ha M, Cosentino M, et al. Long-term storage and recovery of buccal cell DNA from treated cards. Cancer Epidemiol Biomarkers Prev 2006;15:385-388.

51. Atkins D, Reiffen KA, Tegtmeier CL, et al. Immunohistochemical detection of EGFR in paraffin-embedded tumor tissues: variation in staining intensity due to choice of fixative and storage time of tissue sections. J Histochem Cytochem 2004;52:893-901.

52. Zhou H, Yuen PS, Pisitkun T. et al. Collection, storage, preservation, and normalization of human urinary exosomes for biomarker discovery. Kidney Int 2006;69:1471-1476.

53. Ahmad S, Sundaramoorthy E, Arora R, et al. Progressive degradation of serum samples limits proteomic biomarker discovery. Anal Biochem 2009;394:237-242.

54. Isaksson HS, Nilsson TK. Preanalytical aspects of quantitative TaqMan real-time RT-PCR: applications for TF and VEGF mRNA quantification. Clin Biochem 2006;39:373-377.

55. Kauppinen T, Martikainen P, Alafuzoff I. Human postmortem brain tissue and 2-mm tissue microarrays. Appl Immunohistochem Mol Morphol 2006;14:353-359.

56. Rosenling T, Slim CL, Christin C, et al. The effect of preanalytical factors on stability of the proteome and selected metabolites in cerebrospinal fluid (CSF). J Proteome Res 2009;8:5511-5522.
57. Paik S, Kim CY, Song YK, Kim WS. Technology insight: application of molecular techniques to formalin-fixed paraffinembedded tissues from breast cancer. Nat Clin Pract Oncol 2005;2:246-254.

58. Guder WG. Preanalytical factors and their influence on analytical quality specifications. Scand J Clin Lab Invest. 1999;59:545549.

59. Timms JF, Arslan-Low E, Gentry-Maharaj A, et al. Preanalytic influence of sample handling on SELDI-TOF serum protein profiles. Clin Chem 2007;53:645-656.

60. Chan KC, Yeung SW, Lui WB, et al. Effects of preanalytical factors on the molecular size of cell-free DNA in blood. Clin Chem 2005;51:781-784.

61. Fiedler GM, Baumann S, Leichtle A, et al. Standardized peptidome profiling of human urine by magnetic bead separation and matrix-assisted laser desorption/ionization time-of-flight mass spectrometry. Clin Chem 2007;53:421-428.

62. Kirk MJ, Hayward RM, Sproull M. Non-patient related variables affecting levels of vascular endothelial growth factor in urine biospecimens. J Cell Mol Med 2008;12:1250-1255.

63. Kueltzo LA, Wang W, Randolph TW, et al. Effects of solution conditions, processing parameters, and container materials on aggregation of a monoclonal antibody during freeze-thawing. J Pharm Sci 2008;97:1801-1812.

64. Ginocchio CC, Wang XP, Kaplan MH, et al. Effects of specimen collection, processing, and storage conditions on stability of human immunodeficiency virus type 1 RNA levels in plasma. J Clin Microbiol 1997;35:2886-2893.

65. Braakhuis BJM, Tabor MP, Kummer JA, et al. A genetic explanation of slaughter's concept of field cancerization: evidence and clinical implications. Cancer Res 2003;63:1727-1730.

66. Deng G, Lu Y, Zlotnikov G, et al. Loss of heterozygosity in normal tissue adjacent to breast carcinomas. Science 1996;274:2057-2059.

67. Mojica WD, Stein L, Hawthorn L. An exfoliation and enrichment strategy results in improved transcriptional profiles when compared to matched formalin fixed samples. BMC Clin Pathol 2007;7:7.

68. Umar A, Dalebout JC, Timmermans AM, et al. Method optimisation for peptide profiling of microdissected breast carcinoma tissue by matrix-assisted laser desorption/ionisation-time of flight and matrix-assisted laser desorption/ionisation-time of flight/time of flight-mass spectrometry. Proteomics 2005;5:26802688.

69. Breit $\mathrm{S}$, Nees M, Schaefer U, et al. Impact of pre-analytical handling on bone marrow mRNA gene expression. Br J Haematol 2004;126:231-243.

70. Coudry RA, Meireles SI, Stoyanova R, et al. Successful application of microarray technology to microdissected formalin-fixed, paraffin-embedded tissue. J Mol Diagn 2007;9:70-79.

71. Sanchez-Carbayo M, Saint F, Lozano JJ, et al. Comparison of gene expression profiles in laser-microdissected, nonembedded, and OCT-embedded tumor samples by oligonucleotide microarray analysis. Clin Chem 2003;49:2096-2100. 